\title{
A General Model of Auxiliary Processes in Manufacturing
}

\author{
Mezgár ${ }^{1}$, I., Kovács ${ }^{1}$, G., Gáti ${ }^{1}$, G., Szabó ${ }^{2}$, Cs. \\ ${ }^{1}$ CIM Research Laboratory, Computer and Automation Research Institute, \\ Hungarian Academy of Sciences, E-mail: mezgar@sztaki.hu \\ ${ }^{2}$ G. Tim-Co. Ltd.,1082 Budapest, Hungary, Kisfaludy u. 40.
}

\begin{abstract}
In all sizes of manufacturing enterprises, daily shop-floor activities include a number of tasks not directly related to the main production flow. These complementary activities concern, among others, recycling of used material, productive resource maintenance. The goal of the research work was to capture the knowledge related to these activities and based on this knowledge to generate a general process model applicable for describing recycling-, maintenance- and strategic analysing processes. The paper introduces the general model of an air conditioner refurbishing process and some further applications of this model through a case study.
\end{abstract}

Key words: maintenance, modelling, non-manufacturing process, recycling, simulation.

\section{INTRODUCTION}

Shop-floor processes are becoming more and more complex because of the auxiliary processes that are running parallel with the main production flow (e.g. maintenance, tooling), because of the organisation structures required by the enhanced flexibility, and because of the increasing requirements originating from the green manufacturing concept [4]. Current process models fail to capture and integrate at an appropriate semantic level, all the knowledge elements necessary to describe this complex scenario. Usually, application-dependent models are available at different levels, with different goals [3]. 
CAPP systems provide very detailed descriptions of single operations at design level, production planning and control packages support highly approximated product-oriented process models; production schedulers and simulators support resource-oriented models. In addition, all of these models consider only the main production flow, as activities that run in parallel with the main production process (e.g., maintenance and recycling) are not properly and fully represented in these models [1].

In order to solve the problems originating from this modelling inaccuracy, and parallel give an answer for the market demands, a project named EPSYLON (Enhanced Process Modelling System For Lean Operations Management, No. 25359) was organized. The main objectives of the project were:

1. define, implement and validate a new software for managing process information at the highest possible semantic level, with the possibility of translation it into the STEP standard;

2. integrate the new process model platform with existing scheduling and monitoring packages, in order to improve the current manufacturing operation management;

3. develop new DSS applications that fully exploit the new process model expressiveness, to support recycling, maintenance, simulation and strategic analysis.

The project consortium consisted of innovators, technological partners and end users from six different countries. The expertise of the consortium members cover all scientific, technological, functional and organisational needs, which have been identified as necessary to carry out the project. The paper gives a short overview on the theory of the new modelling approach, the main software modules, and then introduces the results through presenting the different models of a refurbishing process in a railway rolling stock and engineering service firm.

\section{THE EPSYLON SYSTEM}

\subsection{The new modelling approach}

The most important part of the project was the development of the proper representation form of processes that can be applied in different applications.

The process representation is realised through modelling primitives with the following advanced features: 
(a) definition of operation types in abstract terms, capable to describe the different kinds of shop-floor activities (e.g., machining, assembly) independently of the context-dependent and resource-dependent details that characterise execution of single operation instances;

(b) representation of processes as sequences of operations, with parallel, alternative and cyclical paths, directed to a specific goal (e.g., disassembling a returned product, performing a given maintenance procedure);

(c) parametrisation of process and operation definitions, to capture engineering and manufacturing degrees of freedom (e.g., alternative routings for the same component), along with their mutual dependencies and constraints;

(d) description of resources in terms of their structure and variable features (e.g., machine tools configurations), capabilities and independent behaviour (e.g., operation timing, parallel operations execution);

(e) partial or complete specification of process features, in terms of included operations, involved resource instances, location inventory, to build different factory scenarios.

\subsection{System Architecture and Modules}

The enhanced process representation will support decision-making at all levels of factory operations management. With reference to the below architecture scheme, the following software modules have been implemented based on the proposed process model (see Figure 1.).

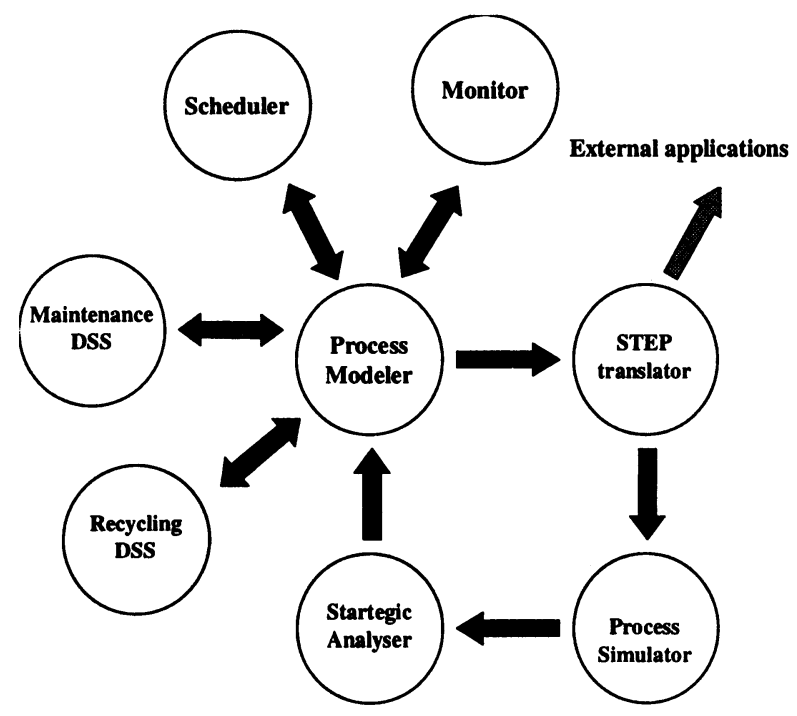

Figure 1. The EPSYLON system architecture. 
Process Modeller. This module provides functions for creating, editing and consultation of process representation schemas based on the EPSYLON model primitives. Process modelling is solved on two levels; conceptual and instance levels. The conceptual level is that dealing with part, operation and resource types, classified according to their general features. The instance level describes the actual factory in terms of existing instances of the above entity types, their specific configurations, relationships, past, current and foreseen states.

STEP translator: translation of EPSYLON process representations into the corresponding STEP representations. The new process model must be capable of both linking to any factory information and control system existing at the user company and also be capable of importing and exporting data to other organisations.

Scheduler (integrated module): integration between the Process Modeller and an existing short-term production planner, extended and improved to take advantage of the new process representation in managing non-standard demand items.

Process Monitor (integrated module): integration between the Process Modeller and an existing process monitoring system, extended and improved to maintain an updated image of the factory in its current status.

Recycling DSS: supports to manage the processes originated from taken-back products, scraps and by-products to be recycled, taking into account all the variable and optional process features (e.g., type and status of the returned product, defects frequency and incidence on the recycling process, disassembly and recovery costs);

Maintenance DSS: supports the user in selecting the appropriate actions (e.g., defining the sequence of operations in reaction to a failure), taking into account general rules and data drawn from experience.

Simulator and Strategic Analyser: development, simulation and evaluation of alternative process scenarios, e.g.: new shop-floor configurations, "green" manufacturing options, introduction of alternative manufacturing, maintenance and inspection methods. Simulation proposes future scenarios in response to a distribution of external events either userdefined or generated by the system on a probabilistic basis.

\subsection{Expected benefits}

The main goal of the project is to produce useful, applicable results for the industry. The expected benefits for the end-users can be classified into three main groups: 
1. Improved management of process data. The system allows enterprises to capture, to understand and to formalise knowledge of their shop-floor processes. The adoption of generalised, family-based primitives will allow a reduction of the representation size, in most cases of above $50 \%$ over traditional structures.

2. Control over complementary operations. The system will support documentation, planning and monitoring of non-manufacturing operations, like recycling and maintenance, as currently is given only for manufacturing ones.

3. Improved decision-making functions. The common process representation will provide a unified, detailed and updated reference for strategic decision-making.

The feasibility of the above improvements, along with the costs and time required for achieving them, was assessed through experimentation of the EPSYLON prototype on user firms in the Consortium.

\section{THE CASE STUDY}

\subsection{The firm}

The firm where one of the pilot implementation took place was a railway rolling stock and engineering service SME. The main activity of the company is to carry out reparation and maintenance on the units of high speed wagons used in international and national inter-city traffic. The periodic maintenance, the reparation of several subsystems of the wagons, like the air-conditioner units, are done by the firm.

The main activities of the firm are usually like a work order, because the reparation and the maintenance jobs are not possible to plan in advance. Unfortunately the periodic maintenance also depends on the traffic, so it is often not done in time, only with deviations. The production is like a small batch production, because the customer needs small quantities. The products are not fixed in advance, the firm works for his customer in a very wide range according to their daily expectations.

\subsection{Description of the air-conditioner refurbishing process}

The main revisions of the different devices are the most precisely determined activity of the company. It has to be taken on all equipment in 
every four years. Although there are several types of these devices, the processes are very similar. For the process model validation the refurbishing of air conditioner has been selected, as this process is rather complex, but at the same time the tasks and their sequences can be described well, and represent the typical refurbishing process at the firm. The process-oriented description of air-conditioner refurbishing can be found in details in Deliverable D02 [2].

This chapter introduces a part of the refurbishing process of the air conditioner by means of the EPSYLON Process Model entities and their relationship. The whole documentation concerns production flow for the air conditioner refurbishing activity and provides a clear representation of what can be done with the primitives of the EPSYLON process model. The process model is suitable for representing all the possible operations (assembly, transformation, disassembly and test), alternative production paths and parts (manufactured, recycled, wasted, etc.) that are involved in a production process flow.

Three basic productive processes have been identified: manufacturing process, disassembly process and recycling process. The relations between these three types of process are the following: the results of a disassembly process are disposed, recycled or further disassembled, the results of recycling processes are used by manufacturing processes, the results of manufacturing processes can feed, after usage, proper disassembly processes.

The disassembly operational schema as it can be seen on Figure 2., is a tree that, starting from the root part, produces some leaf parts. A syntactic exception is given by the possible presence of disassembly alternatives, with or without disassembly joins, and of path alternatives, always with path splits. It has no secondary outputs since unsuccessful cases are explicitly modelled by alternatives. The only types of allowed operations are transformation and disassembly. Leaves can be recovered parts, returned parts (from lower level modules), and wasted materials. Root is always a returned part.

The recycling operational schema is a sequence of transformations (with possible secondary outputs) that produces a recycled part from a recovered part. A syntactic exception is given by the possible presence of path alternatives, always accompanied by path splits. The only allowed operations are transformations. Leaf is always a recovered part, root is always a recycled part.

The mixture of these two types of operational schemas can bee identified in the first step of the air conditioner (AC) refurbishing process. Figure 2. shows that $\mathrm{AC}$ is disassembled into six sub-units, while the gas in the $\mathrm{AC}$ starts its recycling process. The refurbishing of each part is described with the use of these symbols in the whole model. 


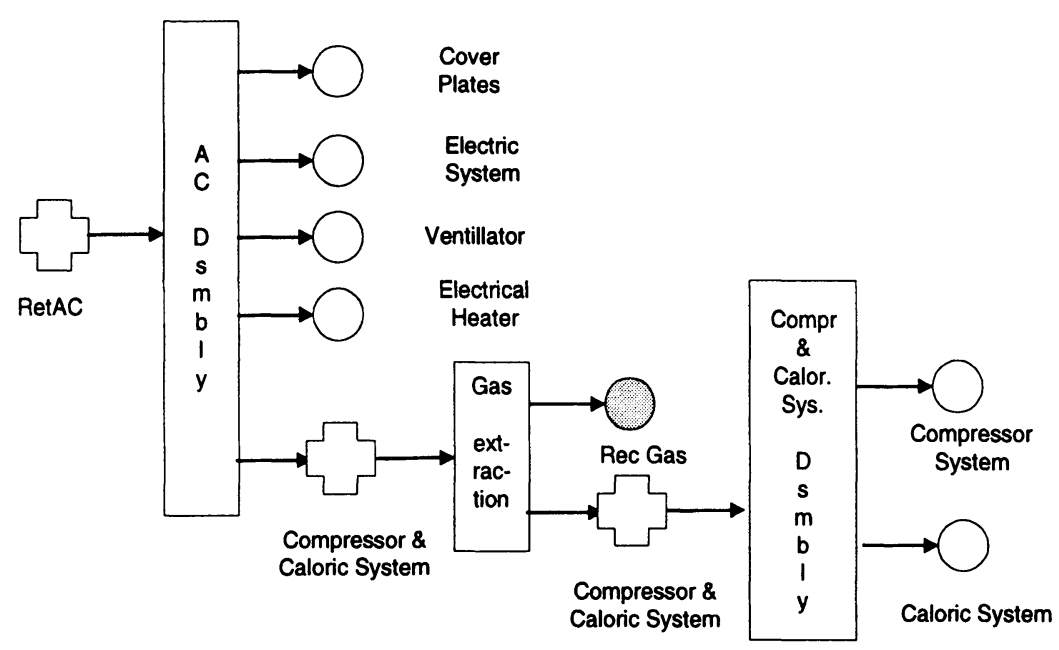

Figure 2. First steps of the refurbishing process of the air-conditioner.

\subsection{Models of the refurbishing process}

\subsubsection{Actual EPSYLON configuration}

As a first step an experimental configuration was installed at the firm. This configuration had more goals, as the validation of the software and the models itself, and besides these ones the user training, collecting more experience were the additional goals.

The configuration that was installed at the firm had to cover the practical needs of the firm, so the following configuration had been tested during the experiment phase:

- Process Modeller (PM),

- Recycling Decision Support System (RDSS),

- Simulator.

The STEP translator was an active part of this configuration as the communication among the simulator and the other modules could be realised through this interface. 


\subsubsection{The models}

The models introduced in this chapter all represent the different phases of the refurbishing process of the electric system, which is a subsystem of the air-conditioner.

The models describing the refurbishing processes are the following, according their sequence of application:

1. operational schema that is the base of modelling. (Fig. 2)

2. general process model (GPM) by using the PM software module (Fig. 3).

3. RDSS model conversion from the GPM.

4. Conversion the GPM into STEP format.

5. Simulation model generation from the STEP format.

The operational scheme is a user-friendly representation of the process, which contains all important process information and can be developed by a person who has little experience in modelling.

The Process Modeller module has the most important role in the applications, as this is the base for all applications. The PM is generated from the operational scheme. It is also investigated from the aspect, how it could be used to serve process data for other applications as the EPSYLON.

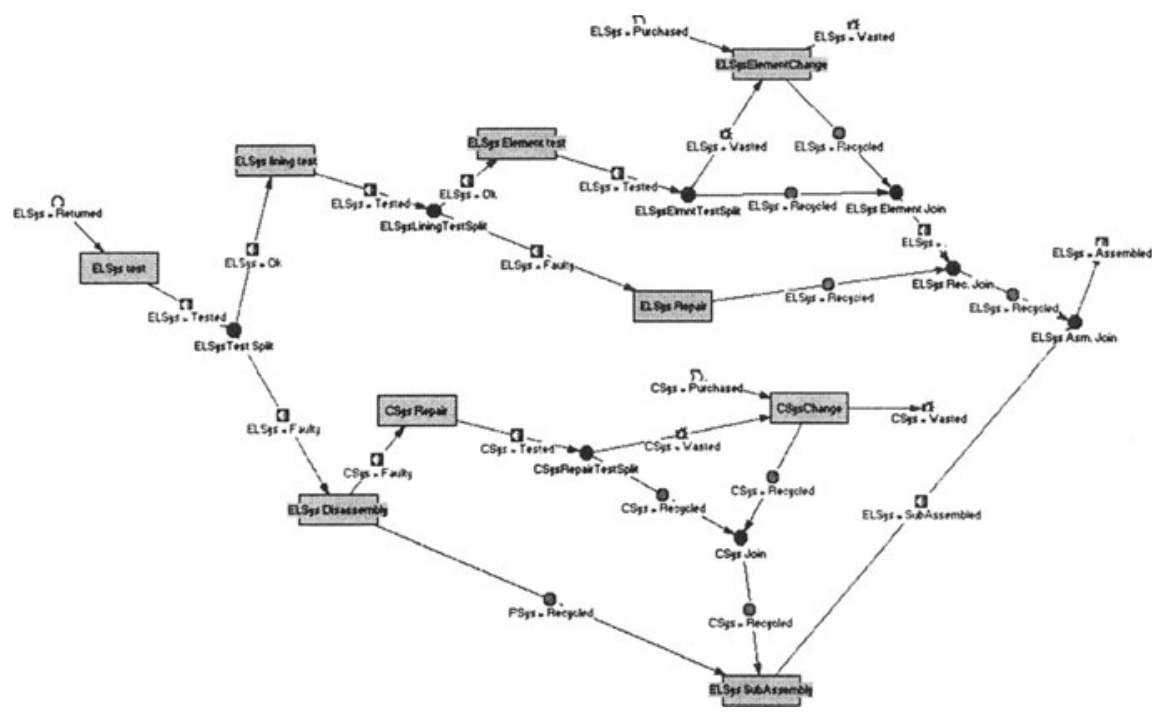

Figure 3. The ELSys process model 
The connection between PM and RDSS is the following; the process model can describe the different phases of the life cycle. The RDSS is appropriate to follow and handle these phases and it is possible to predict (on a certainty level) some of the unscheduled corrective actions. By checking and processing (if it is needed) these failures and repair them with the planned maintenance action, can save a lot of time and can extend the MTBF (mean time between failure) of the actual unit.

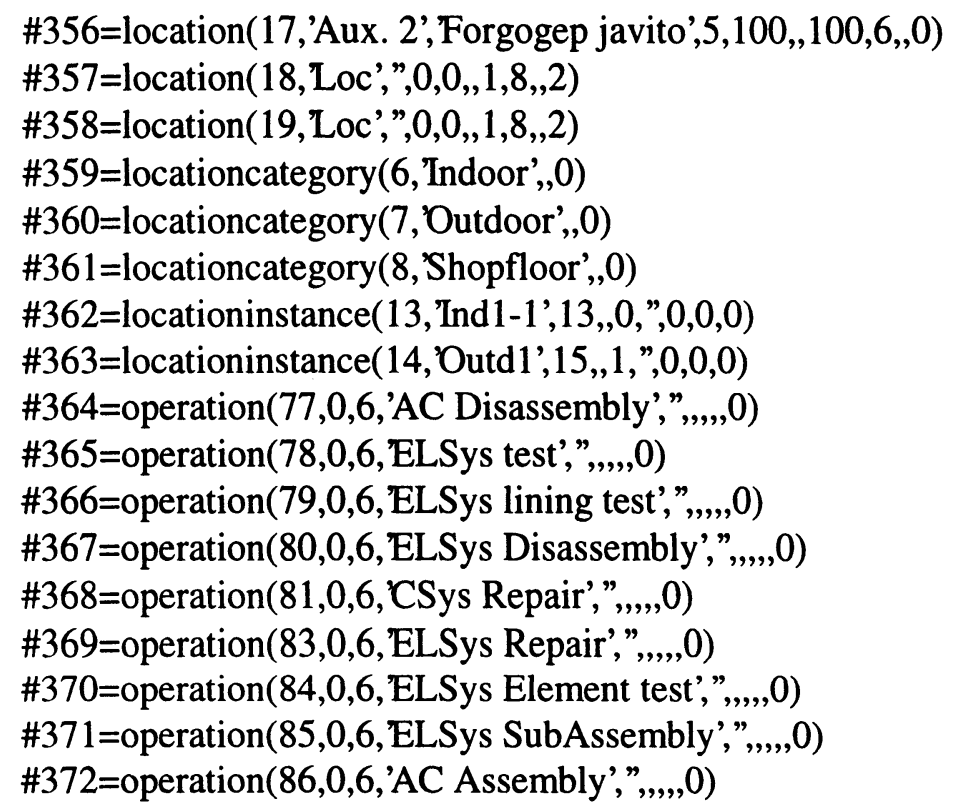

Figure 4. The STEP representation of ELSys model

The STEP "module" represents the openness of the EPSYLON system. Through this module it is possible to export/import process description to/from other systems. In Figure 4 a part of the converted Process Model of the electronic system can be seen.

The simulation model is build from the STEP representation in the Taylor simulation system. The simulation was used in the pilot case for resource analyses, for definition of bottle - necks and initial schedule generation.

The process model can be modified easily according to the actual needs. The possible developments are; to create a more complex process by adding more subprocesses, develop a deeper model by giving more details. The increased usability of the model - closer to real-life application - results increasing motivation of the user.

An important aspect was the user's reaction during the project. Having seen the computer model and the possibilities (e.g. in use of RDSS) user started to show more enthusiasm, and in the final model building/user 
training phase they were nearly fully convinced on the usefullness of the software system.

\section{CONCLUSIONS}

The paper introduced a new modelling approach to represent nonmanufacturing processes in a general way. This model can be applied by different application oriented programs increasing communication/data exchange speed among applications and decreasing the risk of multiple data handling, and the time of multiple data input. The set of decision systems based on this model offers help among others, in the management of maintenance, recycling and monitoring processes. The general model supports the analysis of these processes as well.

At user sites the practical results are that the system allows enterprises to capture, understand and formalise knowledge of their shop-floor processes, at a proper detail level and with the amplest possible scope. This radically improves all activities inside (and in many cases also outside) the firm. By using the information system based on the formalised process descriptions the work of both the managers and the workers on the shop floor will be more effective.

\section{REFERENCES}

[1] Bonfatti F., Monari P. D., Paganelli P. A rule-based manufacturing modelling system, International Journal of Computer Applications in Technology (IJCAT), Vol 10, 1-2, 1997.

[2] EPSYLON Consortium. Deliverable D02 - Process Model, June, 1998.

[3] Kurbel K., Rautenstrauch C. Integrated Planning of Production and Recycling Processes, MIM '96 Int. Conference, Leicester (UK). 1996.

[4] Tipnis, V.A. Evolving Issues in Product Life Cycle Design, CIRP Annals, Vol. 42, 1. 1993. 